\title{
Study on Serum Albumin as Prognostic Marker in Dengue
}

\author{
Dr Yogananda Reddy 1 , Dr Roshan $\mathrm{M}_{2}$ \\ PG Resident, Professor 2, Father Muller Medical College, Mangaloe
}

\section{INTRODUCTION}

Dengue is the most rapidly spreading mosquito-borne viral disease in the world. In the last 50 years, incidence has increased 30-fold with increasing geographic expansion to new countries and, in the present decade, from urban to rural settings . An estimated 50 million dengue infections occur annually worldwide. ${ }^{1}$ There are four virus serotypes, which are designated as DEN-1, DEN-2, DEN-3 and DEN-4. At present DEN1 and DEN2 serotypes are widespread in India ${ }^{2}$. Dengue is transmitted by the bite of female Aedes mosquito .The dengue virus enters via the skin while an infected mosquito is taking a blood meal. During the acute phase of illness the virus is present in the blood and its clearance from this compartment generally coincides with defervescence. Humoral and cellular immune responses are considered to contribute to virus clearance via the generation of neutralizing antibodies and the activation of CD4+ and CD8+ T lymphocytes ${ }^{3}$.

In addition, innate host defence may limit infection by the virus. After infection, serotype specific and crossreactive antibodies and $\mathrm{CD} 4+$ and $\mathrm{CD} 8+\mathrm{T}$ cells remain measurable for years. Plasma leakage, haemoconcentration and abnormalities in homeostasis characterize severe dengue ${ }^{4}$. The mechanisms leading to severe illness are not well defined but

.a complex interaction between virus, host immune response and endothelial cells likely impacts the barrier integrity and functions of endothelia cells leading to plasma leakage ${ }^{4}$

Clinical manifestations vary from undifferentiated fever to florid hemorrhage and shock. The clinical presentations depend on age, immune status of the host and the virus strain. It is very important to know early, if the patient is going for any complication. Laboratory data such as Platelet count, Hemoglobin and PCV are presently used to asses and to manage complication of dengue, such as dengue hemorrhagic fever and dengue shock syndrome. Severe Dengue will be having plasma leakage which may result in low serum Albumin 5 .

Methods

This is descriptive observational retrospective study of secondary data obtained from hospital records of Father Muller medical college Mangalore which is tertiary referral center. All male and female patients aged above 18 years who were tested positive either for NS1 antigen or positive for IGM dengue were selected for the study. Subjects with history of, chronic liver disease.Nephrotic syndrome and chronic alcoholics are excluded from the study. This study was approved by institutional Ethics committee

Name Age, Sex, Address and laboratory values such as HB, PCV, Platelet count, Serum Albumin, serum urea and serum creatinine were entered in the proforma. Clinical parameters such asPulse,BP,Respiratory Rate and signs of severe dengue such as nasal bleeding, oral bleeding, gastrointestinal bleeding and any evidence of internal bleeding was entered in proforma. The presence of ascitis and pleural effusion was entered in proforma. Those patients with severe dengue were assigned to Group- 1 and those with uncomplicated dengue were assigned to Group-2.serum albumin was compared, between Group-1and group-2.

The Wilcoxan method (Nonparametric) and student $t$ test (parametric) were used for statistical analysis Descriptive statistics were compared using the chi-square test for variables of gender, age, clinical forms of dengue and laboratory parameters.

\section{Results}

Results of 100 patients with clinical and laboratory diagnosis of dengue fever were analysed. Among the 100 people studied 92 subjects were uncomplicated dengue (Group-1), and 8 were complicated dengue (Group-2).

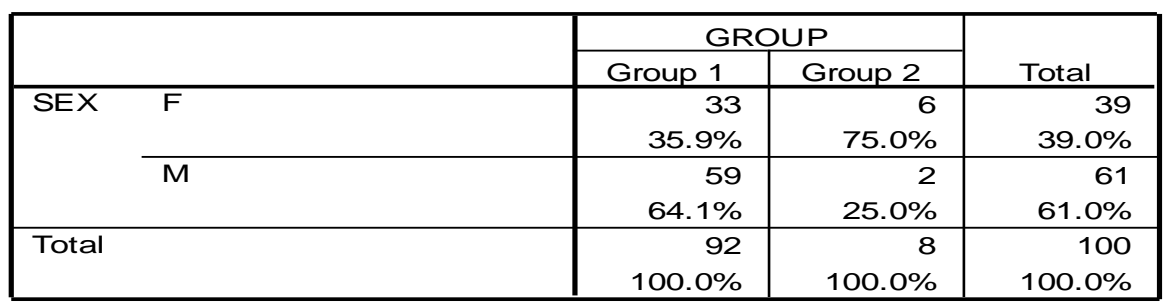


Among the study subjects females constituted 39(39\%) and males constituted 61 subjects $(61 \%)$

\begin{tabular}{|c|c|c|c|c|c|c|c|c|c|}
\hline & GROUP & $\mathrm{N}$ & Minimum & $\begin{array}{l}\text { Maximu } \\
\mathrm{m}\end{array}$ & Mean & $\begin{array}{l}\text { Std. } \\
\text { Deviation }\end{array}$ & Median & $\begin{array}{l}\text { Mannwhi } \\
\text { tney test } \\
\text { Z value }\end{array}$ & $\mathrm{p}$ value \\
\hline \multirow[t]{3}{*}{ HB D1 } & Group 1 & 92 & 9.4 & 18.0 & 13.92 & 1.86 & 14.15 & .23 & .819 \\
\hline & Group 2 & 8 & 12.0 & 16.3 & 13.84 & 1.64 & 13.55 & & NS \\
\hline & Total & 100 & 9.4 & 18.0 & 13.92 & 1.84 & 14.10 & & \\
\hline \multirow[t]{3}{*}{ HB D2 } & Group 1 & 92 & 9.1 & 16.9 & 13.60 & 1.74 & 13.75 & 1.16 & .245 \\
\hline & Group 2 & 8 & 9.6 & 16.0 & 12.88 & 1.81 & 12.85 & & NS \\
\hline & Total & 100 & 9.1 & 16.9 & 13.54 & 1.75 & 13.65 & & \\
\hline \multirow[t]{3}{*}{ HB D3 } & Group 1 & 92 & 8.5 & 16.6 & 13.59 & 1.73 & 13.65 & 1.40 & .160 \\
\hline & Group 2 & 8 & 7.2 & 15.8 & 12.38 & 2.46 & 12.45 & & NS \\
\hline & Total & 100 & 7.2 & 16.6 & 13.50 & 1.81 & 13.60 & & \\
\hline \multirow[t]{3}{*}{ HB D4 } & Group 1 & 92 & 8.4 & 16.4 & 13.55 & 1.72 & 13.60 & 1.72 & .085 \\
\hline & Group 2 & 8 & 6.3 & 15.0 & 12.14 & 2.58 & 12.30 & & NS \\
\hline & Total & 100 & 6.3 & 16.4 & 13.44 & 1.83 & 13.60 & & \\
\hline \multirow[t]{3}{*}{ HB D5 } & Group 1 & 92 & 8.5 & 17.4 & 13.48 & 1.76 & 13.55 & 1.02 & .309 \\
\hline & Group 2 & 8 & 7.6 & 16.0 & 12.61 & 2.45 & 12.70 & & NS \\
\hline & Total & 100 & 7.6 & 17.4 & 13.41 & 1.83 & 13.35 & & \\
\hline
\end{tabular}

AS shown in above tableHemoglobin which was done dialy was compared between group1 and Group-2.Group-2, subjects did not show any statistically significant increase in Hemoglobin.

\begin{tabular}{|c|c|c|c|c|c|c|c|c|c|}
\hline & GROUP & $\mathrm{N}$ & Minimum & Maximum & Mean & $\begin{array}{l}\text { Std. } \\
\text { Deviation }\end{array}$ & Median & $\begin{array}{l}\text { Mannwhit } \\
\text { ney test } \mathrm{Z} \\
\text { value }\end{array}$ & $\mathrm{p}$ value \\
\hline \multirow[t]{3}{*}{ PCV D1 } & Group 1 & 92 & 12.6 & 59.4 & 40.41 & 6.69 & 41.15 & .06 & .954 \\
\hline & Group 2 & 8 & 34.5 & 49.7 & 40.69 & 5.15 & 39.30 & & NS \\
\hline & Total & 100 & 12.6 & 59.4 & 40.43 & 6.56 & 40.90 & & \\
\hline \multirow[t]{3}{*}{ PCV D2 } & Group 1 & 92 & 28 & 53 & 40.48 & 5.62 & 41.15 & 1.50 & .134 \\
\hline & Group 2 & 8 & 32 & 45 & 37.88 & 3.68 & 37.20 & & NS \\
\hline & Total & 100 & 28 & 53 & 40.27 & 5.52 & 40.95 & & \\
\hline \multirow[t]{3}{*}{ PCV D3 } & Group 1 & 92 & 26 & 468 & 44.96 & 44.90 & 41.00 & 1.51 & .132 \\
\hline & Group 2 & 8 & 21 & 45 & 36.50 & 7.08 & 37.40 & & NS \\
\hline & Total & 100 & 21 & 468 & 44.28 & 43.15 & 40.55 & & \\
\hline \multirow[t]{3}{*}{ PCV D4 } & Group 1 & 92 & 25.7 & 50.4 & 39.98 & 5.07 & 40.70 & 1.37 & .170 \\
\hline & Group 2 & 8 & 22.9 & 46.5 & 36.70 & 6.63 & 37.45 & & NS \\
\hline & Total & 100 & 22.9 & 50.4 & 39.72 & 5.24 & 40.10 & & \\
\hline \multirow[t]{3}{*}{ PCV D5 } & Group 1 & 92 & 26.1 & 50.6 & 39.96 & 5.12 & 41.00 & 1.39 & .166 \\
\hline & Group 2 & 8 & 24.9 & 47.0 & 37.01 & 6.18 & 36.90 & & NS \\
\hline & Total & 100 & 24.9 & 50.6 & 39.72 & 5.24 & 41.00 & & \\
\hline
\end{tabular}

As shown in above table PCV was compared between Group-1 and Group-2.Group-2 did not show any statistically significant elevation compared to Group-1. 
Study On Serum Albumin As Prognostic Marker In Dengue

\begin{tabular}{|l|l|l|l|l|l|l|l|l|l|}
\hline & GROUP & $\mathrm{N}$ & Minimum & Maximum & Mean & $\begin{array}{l}\text { Std. } \\
\text { Deviation }\end{array}$ & Median & $\begin{array}{l}\text { Mannwhitney } \\
\text { test Z value }\end{array}$ & $\begin{array}{l}\text { p } \\
\text { value }\end{array}$ \\
\hline PLAT D1 & Group 1 & 92 & 5000 & 208000 & 81633.70 & 55923.62 & 82000.00 & .98 & .325 \\
\hline & Group 2 & 8 & 5000 & 146000 & 62250.00 & 64061.91 & 23500.00 & & NS \\
\hline & Total & 100 & 5000 & 208000 & 80083.00 & 56505.20 & 76500.00 & & \\
\hline PLAT D2 & Group 1 & 92 & 5000 & 251000 & 74743.48 & 48288.98 & 72000.00 & 1.21 & .227 \\
\hline & Group 2 & 8 & 7000 & 121000 & 51125.00 & 44337.79 & 34000.00 & & NS \\
\hline & Total & 100 & 5000 & 251000 & 72854.00 & 48206.48 & 71000.00 & & \\
\hline PLAT D3 & Group 1 & 92 & 8000 & 175000 & 75114.13 & 39043.38 & 79000.00 & 1.08 & .280 \\
\hline & Group 2 & 8 & 10000 & 98000 & 57875.00 & 36556.95 & 54500.00 & & NS \\
\hline & Total & 100 & 8000 & 175000 & 73735.00 & 38958.83 & 78000.00 & & \\
\hline PLAT D4 & Group 1 & 92 & 10000 & 211000 & 86347.83 & 46033.78 & 86500.00 & .44 & .656 \\
\hline & Group 2 & 8 & 22000 & 146000 & 77375.00 & 41507.10 & 80500.00 & & NS \\
\hline & Total & 100 & 10000 & 211000 & 85630.00 & 45559.52 & 86500.00 & & \\
\hline PLAT D5 & Group 1 & 92 & 20000 & 980000 & 126088.04 & 103102.34 & 101000.00 & .30 & .765 \\
\hline & Group 2 & 8 & 48000 & 168000 & 105250.00 & 39766.28 & 102500.00 & & NS \\
\hline & Total & 100 & 20000 & 980000 & 124421.00 & 99575.05 & 101500.00 & & \\
\hline
\end{tabular}

As shown in above table platelets were compared between Group-1 and Group-2.Group-2 patients had lower platelet count compared to group-1, but was not statistically significant.

Serum albumin was also compared between group1 and group-2.Serum albumin was low in Group-2, compared to Group-1 which is statistically not significant

\begin{tabular}{|c|c|c|c|c|c|c|c|c|c|}
\hline & GROUP & $\mathrm{N}$ & Minimum & Maximum & Mean & $\begin{array}{l}\text { Std. } \\
\text { Deviation }\end{array}$ & Median & $\begin{array}{l}\text { Mannwhit } \\
\text { ney test Z } \\
\text { value }\end{array}$ & $\mathrm{p}$ value \\
\hline \multirow{3}{*}{$\begin{array}{l}\text { S.ALBUMIN } \\
\mathrm{mg} / \mathrm{dl}\end{array}$} & Group 1 & 92 & 2.4 & 4.8 & 3.77 & .51 & 3.80 & 1.04 & .296 \\
\hline & Group 2 & 8 & 2.5 & 4.4 & 3.55 & .64 & 3.55 & & NS \\
\hline & Total & 100 & 2.4 & 4.8 & 3.76 & .52 & 3.80 & & \\
\hline
\end{tabular}

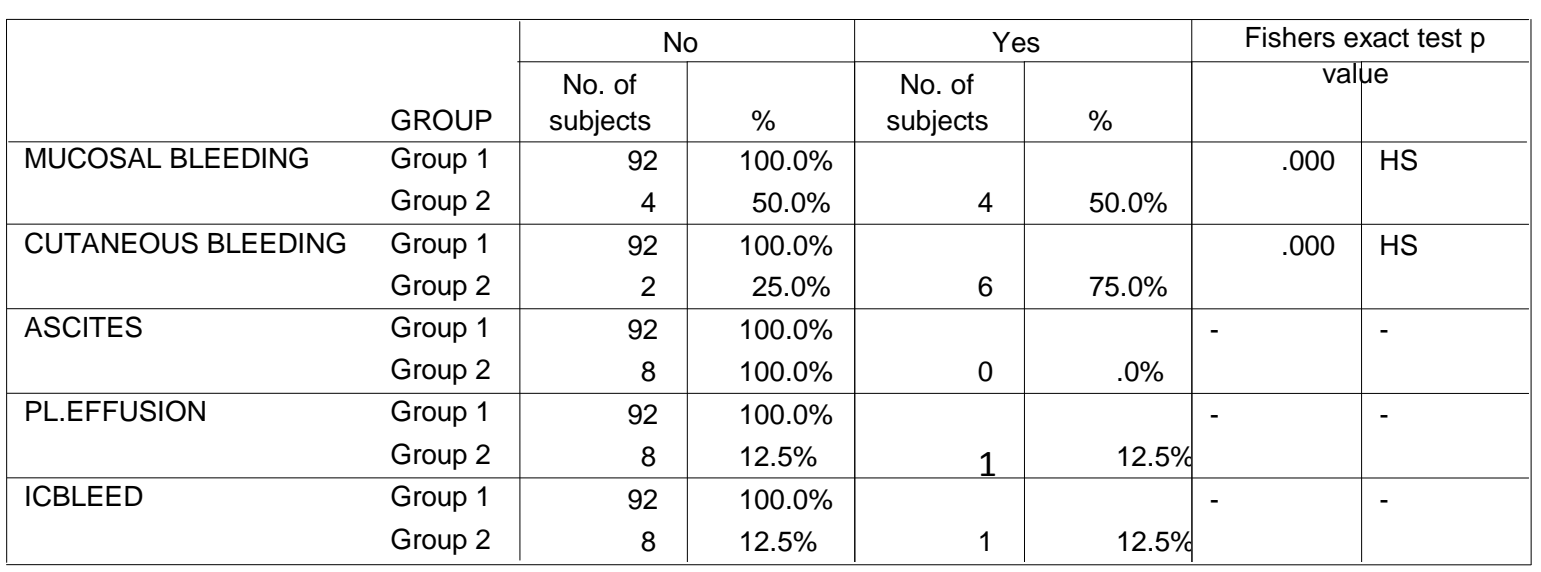

Among Group-2,4 patients had mucosal bleeding, 6 patients had cutaneous bleeding, 1 patient had pleural effusion and intra-cranial bleed was present in1 patient

\section{Discussion}

Dengue is the most common arthropod borne viral disease prevalent in this part of the world. Dengue can either subside spontaneously and may progress to complications. although can be diagnosed early with presently available tests. It is difficult to predict early whether particular person will go to complication ${ }^{6,7}$.

A complex interaction between virus, host immune response and endothelial cells likely impacts the barrier integrity and functions of endothelia cells leading to plasma leakage hypoalbuminemia. Numbers of studies have found relation between severe dengue and albumin levels ${ }^{8,9}$.

This study done retrospectively and included 100 subjects. Among the 100 subjects studied, there were 92 uncomplicated cases and 8 complicated dengue cases. Males were more in this study which constituted about 69 patients and females about 31 patients. There is no preponderance for any sex in the literature severe dengue 
has some of characteristic biochemical and haematological abnormalities. In our study the hematocrit and haemoglobin was elevated on $3^{\text {rd }}$ day in Group-2 patients compared to Group-1 but was not statistically significant.

Serum albumin was compared between group-2 and Group-1.Though serum albumin was comparatively low inGroup-2 compared to Group-1, but was statistically not significant.

In study done by Luis Angel Villar-centeno et al serum albumin above 4 was associated with lower risk of DHF,but that value is not statistically not evaluated ${ }^{10}$.

Serial monitoring of serum albumin may help in monitoring if particular person will go to complication.

\section{References}

[1]. Organisation WH, Editor. Dengue and dengue hemorrhagic fever. Who Geneva;2000

[2]. Dengue Net in India. Weekly Epidemiological Record 2004; 79:57-6.

[3]. Brio CA, Albuquerque mf, Lucena-Silva N. Plasma leakage detection in severe dengue: When serum albumin quantification plays a role Rev SocBras Med Trop 2007; 40:220-3

[4]. Srikiatkhachorn A. Plasma leakage in dengue hemorrhagic fever. ThrombHaemost 2009; 102:1042-49.

[5]. Lee IK, Liu JW, Yang ,.Clinical Characteristics, Risk factors, and outcomes in Adults experiencing Dengue Hemorrhagic Fever Complicated with Acute Renal Failure.Am.J Trop Med Hyg.80;2009:651-5.

[6]. Raimunda F, Azin G, Goncalvas R P, Pitombara MHDS, Lima D N, Branco I G. Dengue: Profile of haematological and biochemical dynamics. Rev Vrashematolhemostar 2012; 34: 36-41.

[7]. Itha S, Kashyap R, Krishnani N, SaraswathVA,Choudhury et al. Profile of liver involvement in Dengue virus infection. NMJI 2005; 18:18-3.

[8]. Siqueira JB, Martelli CM, Coelho GE, Simplicio AC, Hatch DL. Dengue and dengue hemorrhagic fever, Brazil, 1981-2002. Emerg Infect Dis 2005; 11:48-53.

[9]. Perez JGR, Clark GG, Gubler DJ, Reiter P, Sanders EJ et al. Dengue and Dengue hemorrhagic fever. Lancet 1998; 352: 971-77.

[10]. Villar-centeno LA, Diaz-Quijano FA, Martinej-Vega RA. Biochemical alteration as markers of dengue hemorrhagicfever.Am J Trop Med Hyg 2008; 78: 370-74. 